\title{
Development and definition of a simplified scoring system in patients with multiple myeloma undergoing stem cells transplantation on standard computed tomography: myeloma spine and bone damage score (MSBDS)
}

Alberto Stefano Tagliafico ${ }^{1,2^{*}}$, Liliana Belgioia ${ }^{1,2}$, Alessandro Bonsignore ${ }^{1,2}$, Alessio Signori ${ }^{1}$, Matteo Formica ${ }^{2,3}$, Federica Rossi ${ }^{1}$, Michele Piana ${ }^{4,5}$, Daniela Schenone ${ }^{4}$ and Alida Dominietto ${ }^{2}$

\section{Abstract}

Background: In clinical practice, there is the need to optimize imaging usage in MM patients. Accordingly, the aim of this paper was to develop a simple computed tomography $(C T)$ scoring method for $M M$, able to shorten and simplify the interpretation time with good intra- and inter-reader reliability. This method, named MSBDS (Myeloma Spine and Bone Damage Score) was developed with the final aim to use standard totalbody $\mathrm{CT}$ in the routine practice of MM centres as a complement of standard evaluations in patients undergoing stem cells transplantation.

Methods: We used a widely accepted consensus formation method and literature research during three structured face-to-face meetings specifically designed to combine opinions from a group of experts with proven experience in multiple myeloma care and/or musculoskeletal CT to facilitate the consensus on the field of study topics and the contents of the MSBDS score. Seven practical requisites for the MSBDS score were agreed. A total of $70 \mathrm{MM}$ patients (mean age, 60 years \pm 9.2 [standard deviation]; range, 35-70 years) undergoing total-body CT was included to develop MSBDS scores. Patients data were already stored in the Radiological database for other Research studies IRB approved (054/2019). Readers to test the MSDMS were radiologists and clinicians involved in MM care or expert in bone damage scores with different level of experience in musculoskeletal and total body CT. Readers were blinded to the clinical data of the patients.

Results: The MSBDS scores based on the consensus work described above and literature analysis was finalized. MSBDS is based on an additive scale with assessment of a total body CT with the bone window (Continued on next page)

\footnotetext{
* Correspondence: albertotagliafico@gmail.com

'Department of Health Sciences, University of Genoa, Via A. Pastore 1, 16132

Genoa, Italy

${ }^{2}$ Ospedale Policlinico San Martino, Largo R. Benzi 10, 16132 Genoa, Italy

Full list of author information is available at the end of the article
}

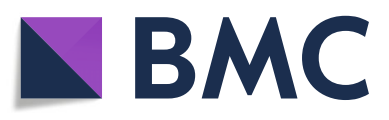

( ) The Author(s). 2020 Open Access This article is licensed under a Creative Commons Attribution 4.0 International License, which permits use, sharing, adaptation, distribution and reproduction in any medium or format, as long as you give appropriate credit to the original author(s) and the source, provide a link to the Creative Commons licence, and indicate if changes were made. The images or other third party material in this article are included in the article's Creative Commons licence, unless indicated otherwise in a credit line to the material. If material is not included in the article's Creative Commons licence and your intended use is not permitted by statutory regulation or exceeds the permitted use, you will need to obtain permission directly from the copyright holder. To view a copy of this licence, visit http://creativecommons.org/licenses/by/4.0/. The Creative Commons Public Domain Dedication waiver (http://creativecommons.org/publicdomain/zero/1.0/) applies to the data made available in this article, unless otherwise stated in a credit line to the data. 


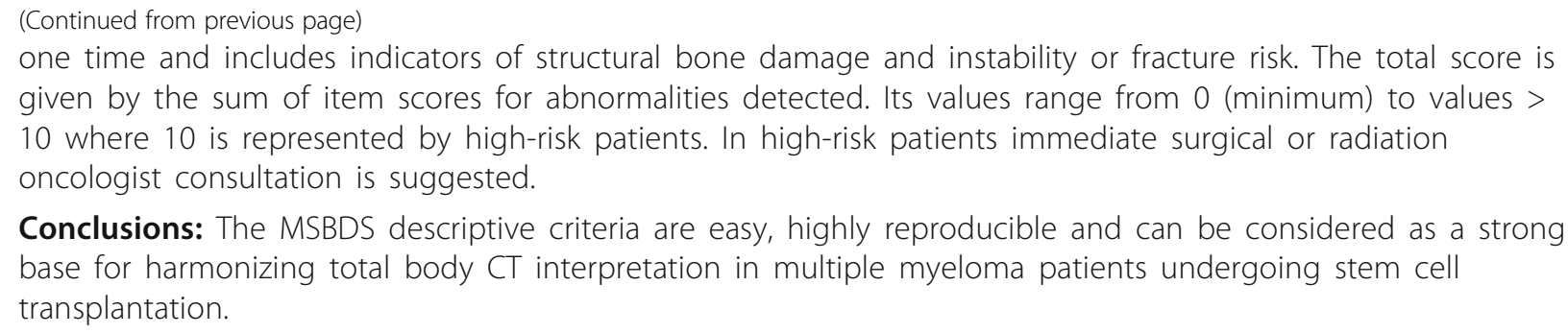

Keywords: Multiple myeloma, Computed tomography, Score, Fracture, Risk, Stem cell transplantation

\section{Introduction}

Abnormal production of monoclonal immunoglobulin $\mathrm{M}$ component of plasma cells and bone marrow increase of plasma cells is the typical characteristic of multiple myeloma (MM). The bone lesions of myeloma are caused by the proliferation of tumour cells from a single clone and the activation of osteoclasts that destroy the bone [1]. Indeed, bone disease reduces patients' quality of life increasing both morbidity and mortality, therefore imaging is crucial in the management of patients with MM. Imaging is important to detect bone lesions requiring immediate start of therapy or follow-up after treatment, to predict the risk of early progression from smouldering MM (SMM) to active disease, to identify sites of extra-medullary disease and to identify sites of bone disease at potential risk of pathologic fractures or neurologic complications [2]. According to recent staging systems for $\mathrm{MM}$, in patients with new diagnosis, a correct treatment approach and evaluation of prognostic factors rely also on lesions identification on Magnetic Resonance Imaging (MRI), Computed Tomography (CT) or PET/CT [3]. Indeed, the role of conventional radiography, the standard of care for many years, is being replaced by more sensitive methods. According to large retrospective studies PET/CT [4] and whole-body lowdose CT (WBLDCT) [5], compared to conventional radiography are able to detect the presence of active disease in up to 25 to $40 \%$ of cases negative at conventional radiography [2]. At diagnosis the incorporation of new imaging modalities (WBLDCT and PET/CT) for accurate diagnostic purposes is recommended with a grade A recommendation [2]. However, there is still considerable heterogeneity in clinical practice regarding imaging usage in MM [2] and the updated International Myeloma Working Group (IMWG) criteria now allow for the use of computed tomography (CT), low-dose whole-body CT, and positron emission tomography with computerized tomography (PET-CT) to diagnose lytic bone disease in MM. However, there is still high variability in the choice between various imaging methods [4] and high variability in image interpretation with suboptimal agreement among readers in CT image interpretation to detect even clinically significant small lytic lesions. Therefore, in clinical practice, there is the need to optimize imaging usage in MM patients. Accordingly, the aim of this paper was to develop a simple CT scoring method for MM, able to shorten and simplify the interpretation time with good intra- and inter-reader reliability. This method, named MSBDS (Myeloma Spine and Bone Damage Score) was developed with the final aim to use standard total-body CT in the routine practice of MM centres as a complement of standard evaluations in patients undergoing stem cells transplantation.

\section{Methods}

We used a widely accepted consensus formation method and literature research, i.e. the nominal group technique (2), during three structured face-to-face meetings specifically designed to combine opinions from a group of experts with proven experience in multiple myeloma care and/or musculoskeletal CT to facilitate the consensus on the field of study topics and the contents of the MSBDS score.

The following requisites for the MSBDS score were agreed: i) easiness in learning by non-expert musculoskeletal radiologists; ii) easiness of use by means of conventional total-body CT scans iii) lack of any requirement for high-end and/ or proprietary technology, i.e. lack of necessity to have low-dose whole body CT or whole body MRI; iv) informativeness in the assessment of bone status compared with standard radiography (skeletal survey), MRI and PET-CT; v) reliability and repeatability for being used to detect small $(>5 \mathrm{~mm}$ ) lytic lesions, monitor treatment efficacy and damage evolution in longitudinal studies; vi) time efficiency to be implemented in daily practice as part of the standard evaluations instead of being an independent diagnostic procedure; and vii) easiness to apply within a busy clinical practice and applicability to a large number of settings with standard $\mathrm{CT}$ available. The agreed requisites for the contents of the MSBDS scoring scale were: i) inclusion of indicators for bone damage; ii) adequate weighing of these items to formulate a scale that most closely reflects the bone involvement in total body CT iii) equal item list for the spine and appendicular skeleton visible on total-body CT. 
Within the nominal group technique meetings, interactive sessions examining the total-body CT of MM patients allowed the board members to build-up a tailored methodology for MM evaluation. Scoring of MM lesions was tailored according to previous literature for spinal involvement in oncological patients [6].

A total of $70 \mathrm{MM}$ patients (mean age, 60 years \pm 9.2 [standard deviation]; range, 35-70 years) undergoing total-body CT with minimum requirements (Table 1) before stem cells transplantations was included to develop MSBDS scores. Patients data were already stored in the Radiological database for other Research studies IRB approved (054/2019).

The staging and the spectrum of bone lesions were sufficiently broad (Table 2) to give an acceptable coverage of the spectrum of severity of bone involvement. According to our standard procedure, all patients signed a written informed consent form, including the permission to use anonimized data for retrospective research purposes, before CT examination.

Readers to test the MSDMS were radiologists and clinicians involved in $\mathrm{MM}$ care or expert in bone damage scores (A.T, L.B, A.B.) with different level of experience in musculoskeletal and total body CT namely: reader 1 (A.T.), > 10 years; reader 2 (A.B.), 3 years; reader 3 (L.B.), 10 years. Readers were blinded to the clinical data of the patients. A focal lesion relevant for MM was defined as a lesion $>5 \mathrm{~mm}$ clinically relevant for diagnosis, prognosis and therapy.

The intra- and inter-observer agreement of the MSBDS score was then calculated. $\mathrm{K}$ statistics were used and $\mathrm{K}$ values were reported as weighed $\mathrm{k}$ with linear weights. 95\% confidence intervals (CI) and standard error were also reported. Agreement was defined on the basis of Fleiss classification: < 0.40 , poor; $0.40-0.59$, moderate; $0.60-0.75$, good; $>0.75$, excellent [7]. Cronbach's alpha was used to assess

Table 1 Minimal and standard Computed Tomography Technical parameters for inclusion

\begin{tabular}{ll}
\hline Number of detector rows* & 16 or more up to 128 \\
\hline Minimum Scan coverage* & Skull base to femur \\
$\begin{array}{l}\text { Tube voltage(kV)/time- } \\
\text { current product (mAs) }\end{array}$ & 120/50-70, adjusted as clinically needed \\
Reconstruction & Sharp, high-frequency (bone) and smooth \\
convolution kernel & $\begin{array}{l}\text { (soft tissue). Middle-frequency kernel for all } \\
\text { images are adjusted by the radiologist as }\end{array}$ \\
& deemed necessary \\
Iterative reconstruction & Yes (to reduce image noise and streak \\
algorithms & artefacts) \\
Thickness & $\leq 5$ mm \\
Multiplanar & Yes (sagittal, coronal and parallel to long \\
Reconstructions (MPRs) & axis of proximal limbs) \\
Matrix, Rotation time, & $128 \times 128,0.5$ s,24 mm per gantry \\
table speed, pith index & rotation, 0.8
\end{tabular}

Table 2 Staging of the $70 \mathrm{MM}$ patients included to develop the MSBDS scores

\begin{tabular}{ll}
\hline & $\begin{array}{l}\text { Number of patients } \\
(n=70)\end{array}$ \\
\hline Durie Salmon Plus & 3 \\
Grade I & 7 \\
Grade II & 51 \\
Grade III & 9 \\
Grade IV & \\
International Staging System & 43 \\
Grade I & 18 \\
Grade II & 9 \\
Grade III & \\
\hline
\end{tabular}

the internal consistency of the method considering values of alpha useful for clinical purposes at least equal to 0.90 [8]. Statistical analysis has been performed with statistical software (MedCalc - version 12.3.0). Finally we correlated MSBDS with MY-RADS score used on CT images to confirm reliability and consistency of the MSBDS compared to MY-RADS.

\section{Results}

The MSBDS scores based on the consensus work described above and literature analysis was finalized as shown in Table 3. MSBDS is based on an additive scale with assessment of a total body CT with the bone window one time and includes indicators of structural bone damage and instability or fracture risk. The total score is given by the sum of item scores for abnormalities detected. Its values range from 0 (minimum) to values $>10$ where 10 is represented by high-risk patients. In highrisk patients immediate surgical or radiation oncologist consultation is suggested.

Spectrum of bone findings is reported in Fig. 1a.

\section{Observer agreements}

Tables 4 and 5 summarise the analysis of inter- and intraobserver tests. Excellent inter-observer and intra-observer agreement was found among the readers. The Cronbach's alpha values for all variables were excellent $(k=0.91)$.

\section{Correlation with MY-RADS score}

A very good correlation between MSBDS and MYRADS score used on CT images was found: $\mathrm{r}=0.816$ with $p<0.001$.

Spectrum of MSBDS score across 70 patients was: high-risk $n=17$; medium risk $n=12$; low-risk $n=41$.

Examples are shown in Fig. 1. 
Table 3 MSBDS (Myeloma Spine and Bone Damage Score). Interpretation: High-risk: > 10: immediate surgical or radiation oncologist consultation. Medium risk: $\geq 5-10$ : possible instability and medium risk of pathologic fracture. Low-risk: $<5$.

\begin{tabular}{ll}
\hline Location & Points \\
\hline Junctional Spine (CO-C2, C7-T2,T11-L1,L5-S1) & 3 \\
Mobile Spine (C3-C6, L2-L4) * only 1 point for semi-rigid (T3-T10) & 2 \\
Collapse/involvement > 50\% & 3 \\
Collapse <50\%* & 2 \\
Posterolateral (facet, pedicle) involvement monolateral & 2 \\
Posterolateral (facet, pedicle) bilateral monolateral & 3 \\
Spinal Canal involvement & 5 \\
Trochanteric region focal lesions < 10 mm & 2 \\
Femoral neck or entire trochanteric region & 5 \\
More 2/3 of bone diameter & 3 \\
Focal lesion > 5 mm at any site* & 1 \\
Diffuse Pattern & $1^{* *}$
\end{tabular}

* Bone abnormalities not sufficient to give high risk scores, if isolated. **1 point for every segment according to MY-RADS [9]
Table 4 Inter-observer agreement among the three readers considering the items of the scoring scale using K value, 95\% confidence intervals and standard error

\begin{tabular}{llll}
\hline & R1-R2 & R1-R3 & R2-R3 \\
\hline $\mathbf{K}$ & 0.87 & 0.91 & 0.85 \\
$\mathbf{9 5 \%}$ C.I. & $0.70-0.95$ & $0.78-1.00$ & $0.73-0.98$ \\
$\mathbf{S E}$ & 0.07 & 0.09 & 0.06 \\
\hline
\end{tabular}

\section{Discussion}

Bone destruction is one of the most prominent features in $\mathrm{MM}$ and represent the main target of analysis included in the MSBDS scoring system. The goal of MSBDS is to provide a semi-quantitative tool to evaluate the status of bone damage and risk of fracture and instability in MM. We must emphasize that the evaluation of the status of the bone in MM is important, but it is only one of the many components used to manage MM patient, and perhaps the most difficult component to judge with good agreement among radiologists and clinicians. Indeed, the low agreement between reader in staging patients affected by multiple myeloma is well

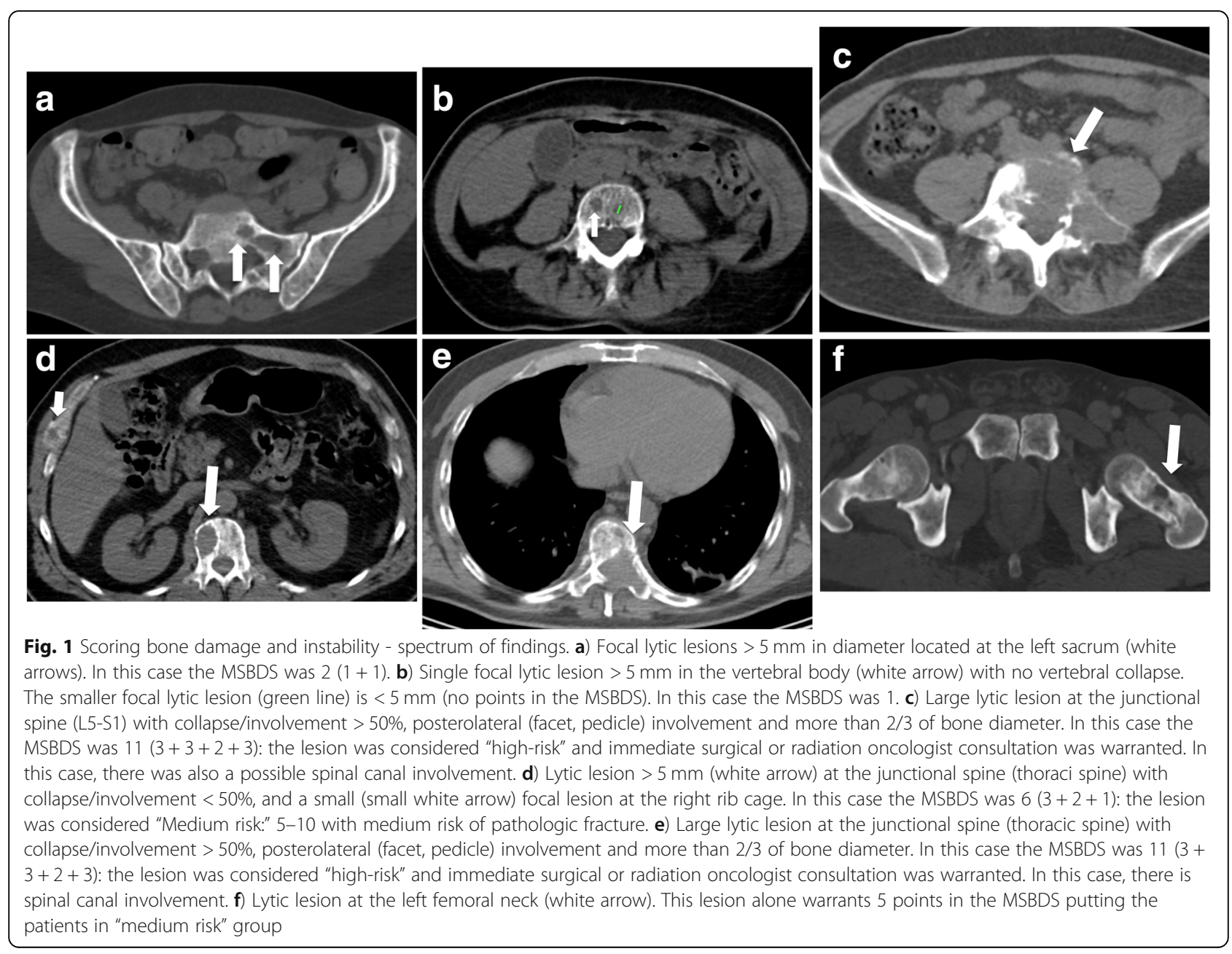


Table 5 Intra-observer agreement among the three readers considering the items of the scoring scale using K value, 95\% confidence intervals and standard error

\begin{tabular}{llll}
\hline & R1 & R2 & R3 \\
\hline $\mathbf{K}$ & 0.84 & 0.81 & 0.85 \\
$\mathbf{9 5 \% ~ C . I . ~}$ & $0.73-0.94$ & $0.73-0.91$ & $0.70-0.93$ \\
SE & 0.09 & 0.06 & 0.05 \\
\hline
\end{tabular}

known in literature [10]: Nanni et al. [9] in the, calculating inter-observer variability with Krippendorff's alpha, found values of 0.56 to 0.58 indicating only moderate agreement for focal lesions. Data reported in the study by Nanni et al. [9] are consistent with recent results of other studies [11] using total body CT and underlines the need of improvement to correctly evaluate patients with bone involvement. Recently, Radiomics was used to improve focal pattern recognition in MM with promising results [11]: using a Radiomics approach it was possible to increase the accuracy of radiological of focal and diffuse pattern of MM on CT. However, the use of very complex imaging techniques such as Radiomics is not possible to be used routinely and it not possible to be implemented in different working environments at the present time. On the contrary, the use of total body CT could be considered feasible in the majority of centres where stem cells transplantations for $\mathrm{MM}$ is available. As for other malignancy, also in multiple myeloma standardization in CT interpretation and scoring of bone lesions is still an unmet need. The evaluation of bone lesions, such as focal lesions, is rapidly gaining importance not only for immediate assessment of dangerous bone lesions (eg. risk of pathological fractures) but also for prognostic purposes. Indeed, the presence of focal lesions is gaining importance because it seems that the presence of focal lesions is linked to poor prognosis [11-15]. In the present study, the MSBDS criteria proved to be highly reproducible, fast and suitable for routine reporting of total body CT in clinical practice in patients with MM undergoing stem cells transplantation. The MSBDS was performed on a relatively high number of patients with relatively advanced stage of disease warranting a high number of bony lesions suitable for the purpose of the study. We demonstrated an "excellent" concordance among the three readers (expert in oncological CT but only marginally trained for MSDBS criteria). This data, as well as the strong correlation with the MY-RADS based score, support the stability of the MSBDS criteria and suggest that this scoring system could be useful to support imaging assessment of totalbody $\mathrm{CT}$ in MM patients. According to recent guidelines on CT in MM $[2,9,10,13-15]$, the MSDBS represent a significant advance in knowledge because it introduces an easy, feasible and fast to assess semi-quantitatively bone lesions in MM. MSBDS has the advantage that it has been tailored on MM patients, differently from previously published scoring systems previously developed in orthopaedic literature for metastatic patients [6]. Indeed, the MSBDS has been developed not only to assess spinal instability, but also to consider bony involvement for prognostic purposes. The introduction of specific items dedicated to proximal femur involvement and to lytic lesion is typical has the aim to be specific for MM patients. There are several advantages of the MSBDS compared to other scoring systems proposed for MM patients such as the MY-RADS [9]. First, the MSDBS can be used on CT images that are far more available than MR images. Second, the MSDBS is very fast and easily reproducible. We are not aware of thorough data on MY-RADS reproducibility and we believe that MSDBS is easier to be used than MY-RADS. We did not assess bone density on CT because a phantom is usually required to calibrate and we would have introduced another source of variability. The use of Radiomics is complex and introduces several critical factors (starting with the necessity to have homogeneous and calibrated acquisition of CT), however Radiomics is a powerful tool and it will be incorporated in the present model if it will result of real clinical usefulness after careful rigorous evaluation. The presence of a very good correlation between MSBDS and MY-RADS score confirmed the reliability and the consistency of the MSBDS compared to MY-RADS. The MSBDS compared to Durie Salmon and Internatonal Staging System it has not the aim to substitute ISS or Durie Salmon, but it has the aim to improve reports and usage of $\mathrm{CT}$ in MM patients. Another advantage of the MSBDS is the relatively low number of analyzed parameters representing a simplification compared to the IMPeTUs criteria for PET or PET/CT. The MSBDS should not increase the burden of data of such a complex disease; therefore we decided to keep this scoring system simple. In addition, the MSDBS could be ready for immediate clinical application indeed it represents a clear improvement of current methods of reporting bone involvement in MM. The MSDBS did not scored different patterns other than focal lesions and diffuse pattern because it seems not clear how other bony patterns visible on $\mathrm{CT}$ are linked to prognosis or to the risk of fracture development. It is possible that with the advent of dual-energy CT different radiological patterns will be confidently determined [16]. Moreover, MSBDS should represent an important tool in order to correctly evaluate the patient's impairment in the medico legal field (e.g.: private health insurances). A prospective clinical validation of MSBDS criteria is underway. We are aware that MSBDS could also be applied in patients with MM even if they are not undergoing stem cells transplantations. 


\section{Conclusions}

In this work we have presented the MSBDS descriptive criteria which are easy, highly reproducible and can be considered as a strong base for harmonizing total body CT interpretation in multiple myeloma patients undergoing stem cell transplantation.

\section{Abbreviations \\ MSBDS: Myeloma Spine and Bone Damage Score; MM: Multiple myeloma; SMM: Smouldering multiple myeloma; MRI: Magnetic resonance imaging; $C T$ : Computed tomography; PET/CT: Positron emission tomography- computed tomography; WBLDCT: Whole-body low-dose CT; MY- RADS: Myeloma Response Assessment and Diagnosis System; IMPeTUs: Interpretation criteria for FDG PET/CT in multiple myeloma}

\section{Acknowledgements}

Not applicable

\section{Authors' contributions}

AT and MF conceived of the present idea. AD supervised the project. AT, FR, $L B$ and $A B$ acquired, analyzed and interpreted the patient data. AT and FR were major contributors in writing the manuscript. MP and DS gave technical support and conceptual advice. All the authors read and revised the manuscript critically, approving the final manuscript.

\section{Funding}

The present work is partially supported by the University of Genova, FRA 2017/2018 granted to Prof. Tagliafico (CdD 4/04/2019).

\section{Availability of data and materials}

The datasets used and/or analyzed during the current study are available from the corresponding author on reasonable request.

Ethics approval and consent to participate

Institutional Review Board approved (054/2019) and written informed consent was acquired from all the patients.

\section{Consent for publication}

Publication is approved by all authors and tacitly or explicitly by the responsible authorities where the work was carried out.

\section{Competing interests}

The authors declare that they have no competing interests.

\section{Author details}

'Department of Health Sciences, University of Genoa, Via A. Pastore 1, 16132 Genoa, Italy. ${ }^{2}$ Ospedale Policlinico San Martino, Largo R. Benzi 10, 16132 Genoa, Italy. ${ }^{3}$ Dipartimento Di Scienze Chirurgiche e Diagnostiche Integrate Disc, University of Genoa, Via A. Pastore 1, 16132 Genoa, Italy. ${ }^{4}$ Dipartimento Di Matematica - Dima, University of Genoa, via Dodecaneso 35, 16146 GENOVA Genoa, Italy. ${ }^{5}$ CNR - SPIN, Genoa, Italy.

Received: 22 January 2020 Accepted: 13 April 2020

Published online: 28 April 2020

\section{References}

1. Jameson JL, Fauci AS, Kasper DL, Hauser SL, Longo JL DL. Harrison's Principles of Internal Medicine. 2018;243.

2. Zamagni E, Cavo M, Fakhri B, Vij R, Roodman D. Bones in Multiple Myeloma: Imaging and Therapy. Am Soc Clin Oncol Educ Book. 2018;38:638-46. https://doi.org/10.1200/EDBK_205583.

3. Filonzi G, Mancuso K, Zamagni E, et al. A Comparison of Different Staging Systems for Multiple Myeloma: Can the MRI Pattern Play a Prognostic Role? AJR Am J Roentgenol. 2017;209(1):152-8. https://doi.org/10.2214/AJR.16. 17219.

4. Siontis B, Kumar S, Dispenzieri A, et al. Positron emission tomographycomputed tomography in the diagnostic evaluation of smoldering multiple myeloma: identification of patients needing therapy. Blood Cancer J. 2015 5(10):e364. https://doi.org/10.1038/bcj.2015.87.
5. Hillengass J, Moulopoulos LA, Delorme S, et al. Whole-body computed tomography versus conventional skeletal survey in patients with multiple myeloma: a study of the International Myeloma Working Group. Blood Cancer J. 2017;7(8):e599. https://doi.org/10.1038/bcj.2017.78.

6. Fisher CG, Dipaola CP, Ryken TC, et al. A novel classification system for spinal instability in neoplastic disease: An evidence-based approach and expert consensus from the spine oncology study group. Spine. 2010. https://doi.org/10.1097/BRS.0b013e3181e16ae2.

7. Landis JR, Koch GG. The Measurement of Observer Agreement for Categorical Data. Biometrics. 1977. https://doi.org/10.2307/2529310.

8. Bland JM, Altman DG. Statistics notes: Cronbach's alpha. BMJ. 1997. https:// doi.org/10.1136/bmj.314.7080.572.

9. Messiou C, Hillengass J, Delorme S, et al. Guidelines for acquisition, interpretation, and reporting of whole-body MRI in myeloma: Myeloma response assessment and diagnosis system (MY-RADS). Radiology. 2019. https://doi.org/10.1148/radiol.2019181949.

10. Nanni C, Versari A, Chauvie $S$, et al. Interpretation criteria for FDG PET/CT in multiple myeloma (IMPeTUs): final results. IMPeTUs (Italian myeloma criteria for PET USe). Eur J Nucl Med Mol Imaging. 2018;45(5):712-9. https://doi.org/ 10.1007/s00259-017-3909-8.

11. Tagliafico AS, Cea M, Rossi F, et al. Differentiating diffuse from focal pattern on Computed Tomography in multiple myeloma: Added value of a Radiomics approach. Eur J Radiol. 2019. https://doi.org/10.1016/j.ejrad.2019. 108739.

12. Hillengass J, Fechtner K, Weber M-A, et al. Prognostic Significance of Focal Lesions in Whole-Body Magnetic Resonance Imaging in Patients With Asymptomatic Multiple Myeloma. J Clin Oncol. 2010;28(9):1606-10. https:// doi.org/10.1200/JCO.2009.25.5356.

13. Rajkumar SV, Dimopoulos MA, Palumbo A, et al. International Myeloma Working Group updated criteria for the diagnosis of multiple myeloma. Lancet Oncol. 2014;15(12):e538-48. https://doi.org/10.1016/S14702045(14)70442-5.

14. Moulopoulos LA, Koutoulidis V, Hillengass J, et al. Recommendations for acquisition, interpretation and reporting of whole body low dose CT in patients with multiple myeloma and other plasma cell disorders: a report of the IMWG Bone Working Group. Blood Cancer J. 2018;8(10):95. https://doi. org/10.1038/s41408-018-0124-1.

15. Rajkumar SV. Evolving diagnostic criteria for multiple myeloma. Hematology 2015. https://doi.org/10.1182/asheducation-2015.1.272.

16. Kosmala A, Weng AM, Krauss B, Knop S, Bley TA, Petritsch B. Dual-energy CT of the bone marrow in multiple myeloma: diagnostic accuracy for quantitative differentiation of infiltration patterns. Eur Radiol. 2018;28(12): 5083-90. https://doi.org/10.1007/s00330-018-5537-5.

\section{Publisher's Note}

Springer Nature remains neutral with regard to jurisdictional claims in published maps and institutional affiliations.

Ready to submit your research? Choose BMC and benefit from:

- fast, convenient online submission

- thorough peer review by experienced researchers in your field

- rapid publication on acceptance

- support for research data, including large and complex data types

- gold Open Access which fosters wider collaboration and increased citations

- maximum visibility for your research: over $100 \mathrm{M}$ website views per year

At BMC, research is always in progress.

Learn more biomedcentral.com/submission 